\title{
Optimization of the maintenance planning of a multi-component system
}

\author{
Issam Mallouk ${ }^{1}$, Badr Abou El Majd ${ }^{1}$, and Yves Sallez ${ }^{2}$ \\ ${ }^{1}$ CeReMAR, LMSA Lab, FSR, Mohamed V University, Rabat, Morocco \\ ${ }^{2}$ UPHV, LAMIH, F-59313 Valenciennes, France
}

\begin{abstract}
The vehicle's maintenance costs, uptime and security are the most important goals for owners and transport companies, but these goals are conflictual and the major cause for delays is related to the maintenance policies. The main objective of transporters is to respond properly to their customer's demands. In order to deal with this competitiveness, transport companies are working to improve the management of their fleets by focusing in particular on vehicle maintenance, which impact the vehicles uptime and generate the most important cost. In addition, a vehicle maintenance policy aims to avoid failures and keep the vehicle up and safe. This objective is reached by ensuring a high reliability; otherwise, an unexpected failure of a component can cause vehicle down and can affect the entire sub-system while generating costs. In this paper, we propose a new maintenance policy based on multi-objective optimization. This problem is solved using the Speed-Constrained Multiobjective Particle Swarm Optimization (SMPSO) for an instance of 18 components and 20 vehicles. First, we give an overview of the existing techniques used for vehicle's maintenance policy, then we present the mathematical model that describes the cost of maintenance and the level of safety. Numerical experiments are presented to demonstrate the efficiency of our approach.
\end{abstract}

\section{Introduction}

In the last decade, maintenance of vehicles in the commercial vehicle industry has received a special attention in order to improve transport solutions and competitiveness [3]. Indeed, in terms of costs, scheduling of maintenance activities permit to save assuredly significant charges. Furthermore, preventive maintenance has the main role in order to avoid vehicle's degradation and especially potential accidents. There are several definitions of maintenance in the literature review. We selected here the one given by AFNOR: "The maintenance maintains or restores a system in a specified state so that it is able to provide a specific service" (AFNOR, N. 2001). Thus, a good maintenance scheduling ensure an efficient and robust decision-making, especially when managing a fleet of vehicles. Actually, in a context with rapid evolution of new technologies, the vehicle is become an active and intelligent actor in transport industry [29]. Vehicle uptime is one of the key parameters to improve transport solutions and competitiveness and it is considered as the most important aspect for commercial vehicles owners who are interested mainly to security and cost of ownership. A vehicle is a multi-components complex system composed of subsystems such as "Electrical supply system", "Braking system" and "engine block" [16. An unexpected failure may cause significant material losses on a certain part or all of the system, or even more dramatically, it may cause human injuries or deaths. The main objective of transporters is to respond properly to their customers demands. Transport companies are working to improve the management of their fleets by focusing in particular on maintenance that impact the vehicles uptime. The aim of preventive maintenance is to avoid failures and keep the system up and running by selecting the right component and decide for change or repair it. The choice is made by following a statistical analysis of the reliability of the components and data reported from the solutions embedded in the vehicle. Nowadays, almost $90 \%$ vehicles are equipped with smarts sensors over wireless communication protocols [5]. The maintenance polices benefits of these technologies by using the vehicle's historical data generated and by remotely analysing and provide diagnosis and maintenance prognosis for driver assistance systems which bring us to the level of e-Maintenance [14] [13. These technological advances also make fault diagnosis and maintenance interventions much more challenging, since these operations require a deep understanding of the entire system [15]. The embedded systems collect the information from the sensors in the format of a raw information. Afterwards, informations are uploaded or synchronized with a remote server over a wireless connection [4]. The authors of [22 [23 have introduced the notion of an 'active product', which although it builds on the concept of product intelligence, it focuses on the ability of a product to trigger actions in its own environment, that is the decision making and execution features. Henceforth, the main challenge is to take benefit from all the collected data based on the analytical capabilities 
of machine learning algorithms and the performance of cloud computing and artificial intelligence [25].

In order to maximize productivity, carriers have a dual interest: perform maintenance without affecting operational uptime and increase productivity by exploiting fleet capacity as optimal as possible. Several strategies of maintenance scheduling were proposed in literature with the aim to develop a maintenance policy by relying on dynamic programming and on a horizon defined previously [5]. All these techniques try to optimize maintenance operations and fleet management but most of them suffer from a lack of real time information. The idea is to use in real time the current information of the components health in order to update the maintenance schedule. The challenge is to keep the maintenance policy valid for the multi-component case, knowing that the components are dependent on each other [19] and each dependency is considered as constraint for another component.

Therefore, the final model turns into an NP-hard problem [16] with a large number of constraints and dependencies to satisfy.

The paper is organized as follows. Section 1 gives a description of the mathematical model. In section2 2 the real instance and the optimization algorithm used to experiment our model are presented. Section 3 presents some numerical results that validate the model and the optimisation process. Finally, section 2 concludes the paper and states some open questions.

\section{The Mathematical model}

In order to maximize productivity, carriers have a dual interest: Perform maintenance without affecting operational uptime and increase productivity by exploiting fleet capacity as optimal as possible.The main aim is to develop a policy which simultaneously optimize maintenance operations and fleet managment. Essentially this work deals with optimization of costs related to component replacements.

\subsection{Notations}

We consider a system Sys has $n$ components, each component $i$ has a replacement $\operatorname{cost} C_{i}$, a replacement time $D_{i}$ and a level of confidence $L C_{i}$ degradable according of time and use. Each level of elementary confidence contributes to the overall level of confidence of the system $L C_{\text {sys. }}$. The idea is that each inspection should check the system confidence level if it has not yet reached the minimum $L C$ and take advantage of the opportunity to replace the other components in dependence [2] and regaining the level $L C_{s y s}$ while minimizing replacement costs and logistical costs associated with downtime.

We summarize in the Table 1 some abbreviations and notations used to explain our mathematical model.

\begin{tabular}{cl}
\hline \hline Abbreviations & Notations \\
\hline$C_{r e m}$ & Remplacement cost \\
$n$ & the number of system components \\
$L C_{s y s}$ & The confidence level of the system \\
$L C$ & The specified confidence level \\
$S y s$ & The system \\
$C_{i}$ & Unit price of the component \\
$D_{i}$ & The replacement period of the componant $i$ \\
\hline
\end{tabular}

Table 1: Abbreviations and Notations

\subsection{Multi-objective optimization}

Almost every real-world problem involves simultaneous optimization of several incommensurable and often competing objectives. While in single-objective optimization the optimal solution is usually clearly defined, this does not hold for multi-objective optimization problems. Instead of a single optimum, there is rather a set of alternative trade-offs, generally known as Paretooptimal solutions. These solutions are optimal in the wider sense that no other solutions in the search space are superior to them when all objectives are considered.

One common approach to multi-objective optimization is the so-called aggregation method, in which the goal is to minimize a single objective, some weighted sum of all objectives. The main advantage of this approach is that any generic optimization algorithm can then be used to minimize this single objective. However, this approach also suffers several drawbacks: it requires some a priori knowledge of the trade- off the decision maker is willing to make between the different objectives. A completely different approach is to use some population based search, and to somehow factorize the efforts by identifying the whole Pareto front at once.

An interesting approach based on game theory was proposed by the authors of [1] [7 [9] to solve multiobjective optimization problems. This approach considers the multi-objective optimization problem as a multi-player co-operative game where each objective function to be optimized is a player in the game. A game is said to be co-operative if the players are able to reach an agreement on strategies. The players are the objective functions, which are ultimately controlled by the decision maker and so can be expected to reach an 'agreement', meaning the game is co-operative. Using the fundamental text on co-operative games [18].

Several multi-objective evolutionary algorithms (MOEAs) have been proposed in literature to solve multi-objective optimization problems [10]. These algorithms are based on different implementation of the Pareto dominance selection and the diversity criterion. In particular, many MOEAs use an archive of solutions, where they maintain the non-dominated solutions ever encountered during the search. Multi-Objective evolutionary algorithms (MOEAs), have demonstrated their ability to do in a flexible and reliable way. 


\subsection{Mathematical Formulation}

The subject of our approach is to find a vector of components to replace or to repair and which is able to give the system a state of good performance. The choice is made by minimizing the replacement cost and by respecting that the confidence level of the system still higher than a specific level (fixed by the decision maker).

In this work, it is assumed that after each maintenance operation where one or several components are replaced, their reliability performances are considered "as good as new". It is also assumed that the reliability performance of the other components is considered unchanged or "as bad as old". Although these assumptions are not necessary they simplify the discussion and do not severely limit the generality of the development. Under these assumptions, the multi-objective optimization problem can be formally stated as follows:

$$
\left\{\begin{array}{l}
\min _{x} \sum_{i=0}^{n} x_{i} *\left[C_{i}+\left(D_{i} * T_{m o}\right)\right] \\
\max _{x} \frac{1}{n} \sum_{i=0}^{n}\left(L C_{i} *\left(1-x_{i}\right)+\left(100 * x_{i}\right)\right) \\
\text { st: }: L C<\frac{1}{n} \sum_{i=0}^{n}\left(L C_{i} *\left(1-x_{i}\right)+\left(100 * x_{i}\right)\right)<100 \\
x \in\{0,1\} .
\end{array}\right.
$$

where $x_{i}$ is a binary variable which indicates the selection of a maintenance operation on the component.

The first objective function aims to select a vector of components to be replaced while minimizing the replacement cost and by respecting that the confidence level of the system still higher than a specific level. The subject of the second objective function is to keep a certain degree of confidence after the replacement of the components, this allows a good performance after the maintenance action. This degree of confidence $L C_{s y s}$ is calculated containing the historical study of the interventions of maintenance, in our case, we summarize this information in a percentage (the sum of perfect elementary confidences $=100 \%$ ). When a need for maintenance is necessary, a maintenance decision rule to select the components to be replaced or repair during this occasion should be defined.

\section{Numerical Results}

\subsection{Provided Data}

This work was conducted in collaboration with STMF company as a part of an industrial project. The real data provided by $S T M F$ is generated manually by an agent who enters the maintenance orders once the maintenance is completed. This helps us to know a complete information after any spare part consumption, but this

\footnotetext{
${ }^{1}$ STMF is a Moroccan company specializing in the transport of sensitive materials (petroleum products, gas, refrigeration, equipment, cabling, etc.)
}

is limited since real time is missing. Several important informations are available.We give in Table 2 a list of spare parts. These data permits to calculate the actual costs of replacement. These informations change regularly, so we create a dynamic database with the component reference as a unique identifier. Table 3 provides us with information on vehicles, odometer and year of entry into service. Also any other information relating to the vehicle in general. In Table 4 we show the most important database, it includes all the information of change and repair of system components. Also it links the two previous lists. We consider this database as a source of historical data.

\begin{tabular}{|l|r|l|l|}
\hline Designation & Unit price & Duration & LC \\
\hline Cushion of air tank & 1205 & 20 & 80 \\
\hline Wiper blade & 61 & 5 & 35 \\
\hline Alu junction reduction & 480 & 15 & 10 \\
\hline Bronze ring & 110 & 5 & 47 \\
\hline Spanner wrench & 75 & 25 & 80 \\
\hline Volvo alternator belt & 388.64 & 60 & 30 \\
\hline Air hose & 120 & 30 & 30 \\
\hline Front oil sensor & 950 & 20 & 20 \\
\hline Volvo front mirror & 576.66 & 15 & 5 \\
\hline Trodume seal & 90 & 10 & 14 \\
\hline Tank brake pad & 761.67 & 30 & 40 \\
\hline
\end{tabular}

Table 2: Data Base list of spare parts

\begin{tabular}{|l|l|l|r|r|} 
Vehicle type & Id & Date of purchase & Registration & Odometer \\
\hline Tractor & 3 & $01-09-2016$ & $35419-A-7$ & 164250 \\
\hline Tractor & 4 & $01-09-2016$ & $35420-A-7$ & 171231 \\
\hline Tractor & 5 & $01-10-2017$ & $49980-A-7$ & 75092 \\
\hline Tractor & 6 & $02-01-2016$ & $47703-A-7$ & 235648 \\
\hline Tractor & 7 & $01-09-2016$ & $35404-A-7$ & 183540 \\
\hline Tractor & 8 & $15-11-2016$ & $36508-A-7$ & 175354 \\
\hline Tractor & 9 & $01-09-2016$ & $35398-A-7$ & 192463 \\
\hline Tractor & 10 & $02-06-2017$ & $44447-A-7$ & 85632 \\
\hline Tractor & 11 & $01-09-2016$ & $35405-A-7$ & 186321 \\
\hline Tractor & 12 & $02-01-2017$ & $41704-A-7$ & 152304 \\
\hline
\end{tabular}

Table 3: Data Base list of vehicles

\begin{tabular}{|c|c|c|c|}
\hline Required work & Spare part & $\begin{array}{l}\text { Date of } \\
\text { maintenance }\end{array}$ & Price \\
\hline Cabin winder to replace & $\begin{array}{l}\text { Volvo spring cab shock } \\
\text { absorber }\end{array}$ & $30-05-2016$ & 940 \\
\hline Cabin carter to be replaced & Front Volvo brake lining & $30-05-2016$ & 940 \\
\hline Alternator belt to replace & Volvo alternator belt & 03-06-2016 & 388.64 \\
\hline Fan belt to replace & Volvo fan belt & 03-06-2016 & 529.41 \\
\hline Wrench wrench to replace & Air horn & 04-06-2016 & 75 \\
\hline Oil sensor to replace & Volvo oil sensor & $12-06-2016$ & 950 \\
\hline $\begin{array}{l}\text { Suspension sensor to } \\
\text { replace }\end{array}$ & Suspension sensor & 21-06-2016 & 1500 \\
\hline
\end{tabular}

Table 4: Data Base list of interventions

\subsection{Estimation of the level of confidences}

A Monte Carlo simulation was used to estimate the level of confidence of each component according to its 
own Weibull parameters. In order to compute the level of confidences, a time unit that varies between 0 and $1,000,000$ units with a step of 1 was chosen, this gives us the simulation over 15 months as shown in figure 1 . The impact of information uncertainty on the estimation of the costs of maintenance replacement is depicted in figure 2 .

The Monte Carlo simulation approach was used in this paper in order to give a numerical solution for real-time system state estimation and prognostics from noisy provided data.

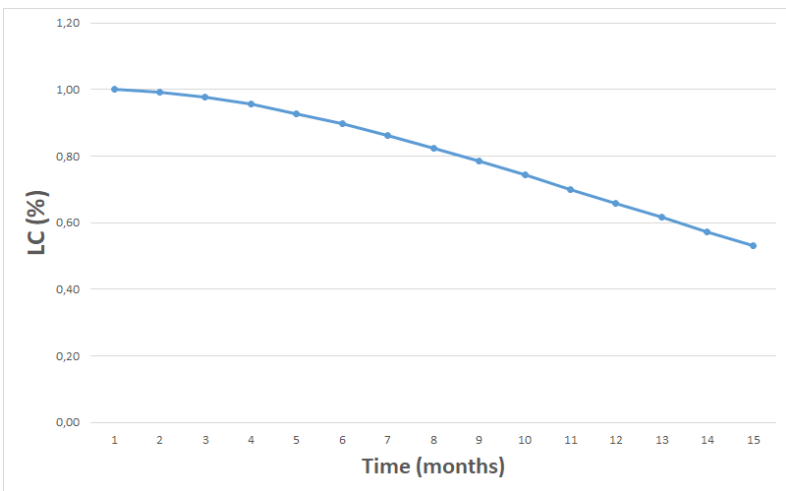

Figure 1: Estimation of the levels of confidences using Monte-Carlo Simulation

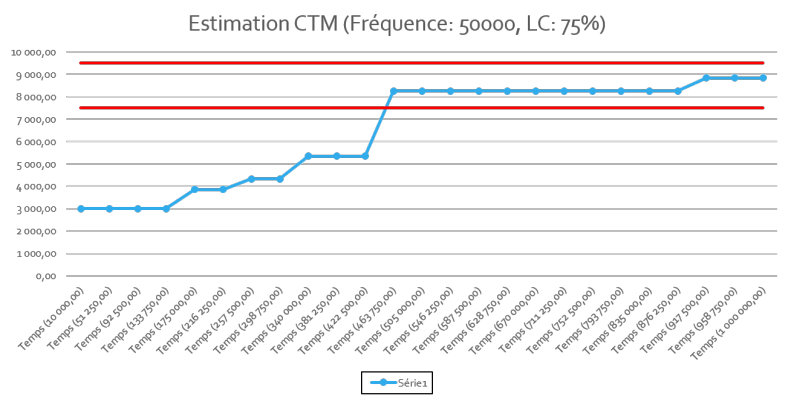

Figure 2: The dependence between time and replacement costs due to Degradation of LC

\subsection{Algorithm}

This section introduces the algorithms that have been used to validate numerically the theoretical framework described above, namely the Speed-Constrained Multi-Objective Particle Swarm Optimization algorithm (SMPSO).

SMPSO [17] is a particle swarm optimization method (bio-inspired) developed from OMOPSO [20. The particle swarm optimization (PSO) algorithms are inspired by the flight of birds while trying to find food. In these algorithms, the population is called swarm. The individuals are called particles, which are "flown
" through space following the best performing particle at that moment. The position of a particle is given by the current values of its parameters, belonging to an orthogonal representation particle's space. As every particle tries to get closer to the current best particle its parameters are changed. The change takes into account both the current global best and the particle's personal best. Based on this change the particle gets a new position and it needs to be evaluated again. After all the particles are evaluated, the new global best particle is selected, the personal bests are updated and the process is restarted. In Multi-objective PSO algorithms there can be multiple global best particles.

SMPSO has been chosen here for its long record of successes, demonstrating its robustness to find a good approximation of the Pareto front [21] [11].

The flowchart below, Figure 3 explain the process of SMPSO.

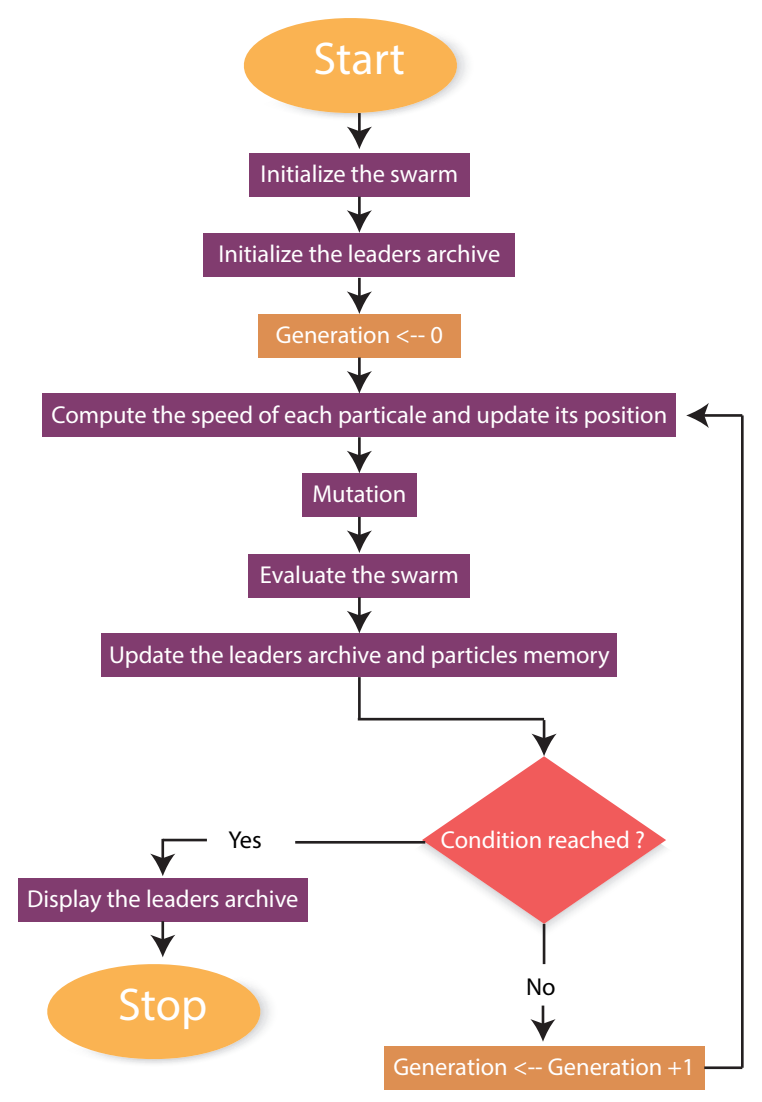

Figure 3: Flowchart of SMPSO algorithm

\subsection{Results}

Several simulations are done to check the CPU time by considering three subcategory of 50 components. Figure 5 shows the CPU time with respect to the components. However, the execution time remains very low. We consider a list of component given by the table 5 . For each component, we generate the level of confidence 
(LC) using Monte-Carlo simulation. Figure 4 gives us the Pareto Front obtained. The two optimal solutions of the objective functions appear in the Pareto front, with an execution time of $19 \mathrm{~s}$ we obtained 15 possible scenarios.

The Pareto front depicted in figure 4 shows the trade-off between minimizing the replacement cost and maximizing confidence level. If the decision maker (DM) wants to minimize the replacement cost no matter the value of confidence level he shall select a solution toward the lower right corner of the graph. Otherwise, if the DM thinks that the confidence level matters, he can choose a compromise in the top lest corner of the graph, resulting in higher confidence level, at the price of a higher cost of replacement.

\begin{tabular}{|c|l|r|c|c|}
\hline Id & Designation & Unit price & Duration & LC \\
\hline $\mathbf{1}$ & Cushion of air tank & 1205 & 20 & 80 \\
\hline $\mathbf{2}$ & Wiper blade & 61 & 5 & 35 \\
\hline $\mathbf{3}$ & Alu junction reduction & 480 & 15 & 10 \\
\hline $\mathbf{4}$ & Bronze ring & 110 & 5 & 47 \\
\hline $\mathbf{5}$ & Spanner wrench & 75 & 25 & 80 \\
\hline $\mathbf{6}$ & Volvo alternator belt & 388.64 & 60 & 30 \\
\hline $\mathbf{7}$ & Fan belt & 529.41 & 40 & 40 \\
\hline $\mathbf{8}$ & Air hose & 120 & 30 & 30 \\
\hline $\mathbf{9}$ & Front oil sensor & 950 & 20 & 20 \\
\hline $\mathbf{1 0}$ & Volvo front mirror & 576.66 & 15 & 5 \\
\hline $\mathbf{1 1}$ & Trodume seal & 90 & 10 & 14 \\
\hline $\mathbf{1 2}$ & Tank brake pad & 761.67 & 30 & 40 \\
\hline $\mathbf{1 3}$ & brake pad & 1398 & 40 & 20 \\
\hline
\end{tabular}

Table 5: List of component

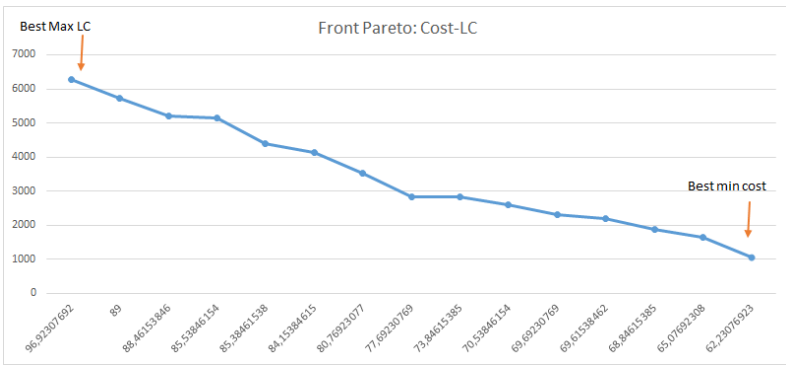

Figure 4: Pareto front Cost-LC

\section{Conclusion}

In this paper, we have proposed a new approach for vehicle's maintenance. The selection of the components to be replaced will be managed by an evolutionary and dynamic mechanism which loop on the subcategories of the components (Engine, braking, suspension ...) each one has its technical specifications which defines its optimal $L C$. On-going and further work will be focusing on integrating the process of simulation and optimization into a single application and try to generate the state of

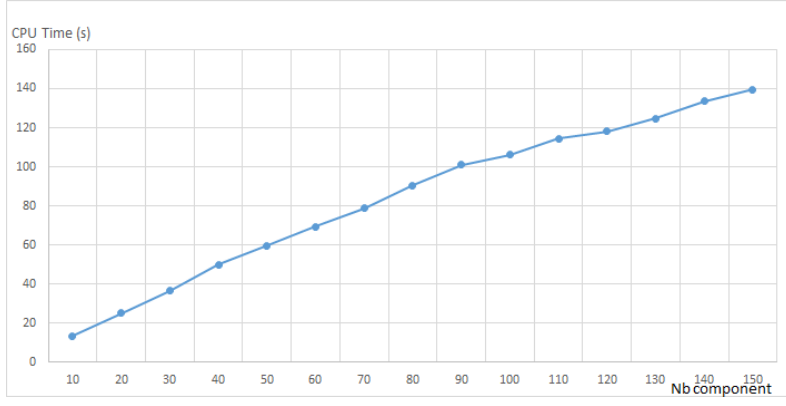

Figure 5: Time CPU evolution

the components of the system by using sensors and embedded solutions while taking advantage of the internet of things. Other MOEAs will be also investigated and compared with SMPSO, and, more importantly, several different instances. One crucial issue is how well this algorithm scales with the problem complexity (number of vehicle and number of components).

\section{References}

[1] B. Abou El Majd, J.A. Désidéri and A. Habbal, " Aerodynamic and structural optimization of a business-jet wingshape by a Nash game and an adapted split of variables". Mécanique Industries, 11(3-4), 209-214, 2010.

[2] Ab-Samat, Hasnida, and S. Kamaruddin. "Opportunistic maintenance $(\mathrm{OM})$ as a new advancement in maintenance approaches: A review".Journal of Quality in Maintenance Engineering 20.2, 98-121, 2014.

[3] S. Alaswad and Y. Xiang," A review on conditionbased maintenance optimization models for stochastically deteriorating system", Reliab. Eng. Syst. Saf., vol. 157, p. 54-63, janv. 2017.

[4] P. Baraldi, F. Cadini, F. Mangili, et E. Zio,“ Modelbased and data-driven prognostics under different available information", Probabilistic Eng. Mech., vol. 32, p. 66-79, 2013.

[5] K. Bouvard, S. Artus, C. Bérenguer, et V. Cocquempot," Condition-based dynamic maintenance operations planning and grouping. Application to commercial heavy vehicles", Reliab. Eng. Syst. Saf., vol. 96, no 6, p. 601-610, juin 2011.

[6] R. Dawid, D. McMillan, and M. Revie,“ Review of Markov models for maintenance optimization in the context of offshore wind", 2015.

[7] R. Duvigneau, B. Abou El Majd and J.A. Désidéri. " Towards a self-adaptive parameterization for aerodynamic shape optimization”. In ESAIM: Proceedings (Vol. 22, pp. 169-174). EDP Sciences, 2008.

[8] K .C. Dey, A. Mishra, and M. Chowdhury, “ Potential of intelligent transportation systems in mitigating adverse weather impacts on road mobility: a review", IEEE Transactions on Intelligent Transportation Systems, vol. 16, no. 3, pp. 1107-1119, 2015. 
[9] J.A. Désidéri, R. Duvigneau, B. Abou El Majd and Z. Tang, " Algorithms for efficient shape optimization in aerodynamics and coupled disciplines”. In 42nd AAAF Congress on Applied Aerodynamics, SophiaAntipolis, France, 2007.

[10] R. Fadil, B. Abou El Majd, H. Rahil, H. El Ghazi and N. Kaabouch, " Multi-objective Optimization Approach for Air Traffic Flow Management”. In: MATEC Web of Conferences, 105, 0005, 2017.

[11] W.F. Fihri, Y. Arjoune, H. El Ghazi, N. Kaabouch and B. Abou El Majd, “ A particle swarm optimization based algorithm for primary user emulation attack detection". In: IEEE consumer communications and networking conference, p. 1-6, 2018.

[12] K. He, L.M. Maillart, and O.A. Prokopyev, "Scheduling Preventive Maintenance as a Function of an Imperfect Inspection Interval", IEEE Transactions on Reliability, vol. 64, no. 3, pp. 983-997, Sep. 2015.

[13] K.T. Huynh, A. Barros, and C. Bérenguer,“ Maintenance decision-making for systems operating under indirect condition monitoring: value of online information and impact of measurement uncertainty", IEEE Trans. Reliab., vol. 61, no 2, p. 410-425, 2012.

[14] S. K. Kinnunen et al., “ Decision making situations define data requirements in fleet asset management", in Proceedings of the 10th World Congress on Engineering Asset Management (WCEAM 2015), 2016, p. 357-364.

[15] A. Le Mortellec, J. Clarhaut, Y. Sallez, T. Berger and D. Trentesaux, “ Embedded holonic fault diagnosis of complex transportation systems”. Engineering Applications of Artificial Intelligence, 26(1), 227240, 2013.

[16] R. Lesobre, “ Modélisation et optimisation de la maintenance et de la surveillance des systèmes multi-composants-Applications à la maintenance et à la conception de véhicules industriels", $P h D$ Thesis, Université Grenoble Alpes, 2015.

[17] A.J. Nebro, J.J. Durillo, J. García-Nieto, C.A. Coello Coello, F. Luna, and E. Alba. " Smpso: A new pso-based metaheuristic for multi-objective optimization ". In 2009 IEEE Symposium on Computational Intelligence in Multicriteria Decision-Making (MCDM 2009), pages 66-73. IEEE Press, 2009.

[18] J. Nash. "Two-person cooperative games”. Econometrica: Journal of the Econometric Society, pages 128-140, 1953.

[19] R.P. Nicolai and R. Dekker, “ A review of multicomponent maintenance models", In : Proc. of
European Safety and Reliability Conference ESREL. 2007.

[20] M. Reyes and C.A. Coello Coello. “ Improving PSObased multi-objective optimization using crowding, mutation and $\epsilon$-dominance ". In C.A. Coello, A. Hernández, and E. Zitler, editors, Third International Conference on Evolutionary MultiCriterion Optimization, EMO 2005, volume 3410 of LNCS, pages 509-519. Springer, 2005.

[21] H. Rahil, B. Abou El Majd, and M. Bouchoum, " Optimized Air Routes Connections for Real Hub Schedule Using SMPSO Algorithm". In : Recent Developments in Metaheuristics, Springer, Cham, p. 369-384, 2018.

[22] Y. Sallez, T. Berger, D. Deneux and D. Trentesaux, " The lifecycle of active and intelligent products: The augmentation concept", International Journal of Computer Integrated Manufacturing, 23, 905-924, 2010.

[23] Y. Sallez, T. Berger and D. Trentesaux, “A stigmergic approach for dynamic routing of active products in fms”, Computers in Industry, 60, 204-216, 2009.

[24] O. Senechal and D. Trenteseaux,“ Spécification d'une méthodologie pour l'aide à la décision dans le cadre de la maintenance basée sur la performance environnementale Application aux systèmes ferroviaires.", Congrès International de Génie Industriel (CIGI), Compiègne, France, mai 2017.

[25] O. Svensson, S. Thelin, S. Byttner, et Y. Fan,“ Indirect Tire Monitoring System - Machine Learning Approach", IOP Conf. Ser. Mater. Sci. Eng., vol. 252, p. 012018 , oct. 2017.

[26] H. C. Vu, P. Do, A. Barros, and C. Bérenguer," Maintenance grouping strategy for multi-component systems with dynamic contexts", Reliability Engineering and System Safety, vol. 132, pp. 233-249, Dec. 2014.

[27] H. Wang, L. Jiao, and X. Yao, "s Two Arch2: An Improved Two-Archive Algorithm for Many-Objective Optimization ”, IEEE Transactions on Evolutionary Computation, vol. 19, no. 4, pp. 524-541, Aug. 2015.

[28] L. Xiao, S. Song, X. Chen, and D. W. Coit,“ Joint optimization of production scheduling and machine group preventive maintenance", Reliability Engineering and System Safety, vol. 146, pp. 68-78, Feb. 2016.

[29] M. Yildirim, X. A. Sun, et N. Z. Gebraeel,“ Sensordriven condition-based generator maintenance scheduling-Part I: Maintenance problem", IEEE Trans. Power Syst., vol. 31, no 6, p. 4253-4262, 2016. 